\title{
Tsafon
}

Revue d'études juives du Nord

$74 \mid 2017$

La Déclaration Balfour, vers l'État d'Israël ?

\section{Un engagement international inachevé}

\section{Emmanuel Persyn}

\section{OpenEdition}

Journals

Édition électronique

URL : https://journals.openedition.org/tsafon/403

DOI : $10.4000 /$ tsafon.403

ISSN : 2609-6420

\section{Éditeur}

Association Jean-Marie Delmaire

\section{Édition imprimée}

Date de publication : 1 décembre 2017

Pagination : 95-104

ISSN : 1149-6630

\section{Référence électronique}

Emmanuel Persyn, « Un engagement international inachevé », Tsafon [En ligne], 74 | 2017, mis en ligne le 31 mai 2018, consulté le 26 juin 2021. URL : http://journals.openedition.org/tsafon/403 ; DOI : https://doi.org/10.4000/tsafon.403 


\title{
Un engagement international inachevé
}

\author{
Emmanuel Persyn*
}

La Déclaration Balfour constituait un succès pour le mouvement sioniste; le premier succès au demeurant dans la mesure où la proposition britannique d'une installation en Ouganda ne marquait certainement pas une reconnaissance politique, mais plutôt une réaction à bon compte aux tragiques pogroms dans l'empire tsariste.

Le 2 novembre 2017, jour de l'envoi de cette déclaration, cela faisait précisément vingt ans que le mouvement sioniste se battait pour une reconnaissance internationale. Le fondateur, Theodor Herzl, s'était épuisé dans de vaines démarches diplomatiques, sans toutefois jamais cesser d'espérer. Ses successeurs à la tête de l'Organisation sioniste mondiale n'avaient guère eu plus de succès.

Il avait fallu attendre les négociations entreprises durant la Première Guerre mondiale pour ouvrir la voie. Dans une conjoncture internationale particulièrement difficile, les grandes puissances avaient même rivalisé pour s'attirer brusquement les grâces d'un mouvement national qu'elles avaient jusqu'alors superbement ignoré.

Les dirigeants sionistes n'étaient pas naïfs au point de se méprendre sur l'approche intéressée de ces responsables politiques, britanniques mais aussi français et allemands. Eux-mêmes étaient rompus à ces pratiques et savaient jouer leur jeu. Tout était question d'opportunités même si cette dimension n'était pas la seule.

Un siècle plus tard, la puissance israélienne peut avoir rejeté dans l'ombre la difficulté et le caractère aléatoire de cette lutte nationale. Il

\footnotetext{
${ }^{*}$ Université de Lille-SHS.
} 
suffit de se rappeler que le cabinet britannique était loin d'être unanime sur l'opportunité de cette déclaration. De la même façon, Nahum Sokolow a dû faire preuve de ténacité pour être reçu par un responsable politique français.

On peut même s'interroger sur l'intérêt de poser la question des conséquences, des traces contemporaines de cette Déclaration Balfour. Le vote de la résolution 181 des Nations unies le 29 novembre 1947 a eu plus d'effets que celle-ci, même si les violences et les guerres ont en définitive beaucoup plus délimité les territoires que cette décision de la communauté internationale.

Alors que reste-t-il de la Déclaration Balfour? On serait tenté de répondre : un document historique de quelques lignes, signifiant par là qu'elle influe bien peu, sinon pas du tout sur l'évolution de la Palestine après la Première Guerre mondiale.

Même si elle a été rédigée dans des termes suffisamment flous pour autoriser toutes les lectures - aucune précision de temps, d'étendue, encore moins de statut n'y figure -, elle s'avère pourtant un enjeu essentiel durant l'Entre-deux guerres. Elle a d'ailleurs été intégrée dans le mandat que la Société des Nations donne à la Grande-Bretagne en 1922.

Les sionistes n'ont en effet cessé d'y faire référence durant le mandat britannique et les Arabes ne l'ont au contraire jamais reconnue. Deux exemples parmi d'autres le montrent. Le 12 août 1937, le vingtième congrès sioniste, appelé à se prononcer sur le plan Peel, « charge l'exécutif de s'opposer à toute atteinte aux droits du peuple juif, garantis internationalement par la Déclaration Balfour et par le mandat ». De leur côté, les Arabes sont déterminés dans leur rejet formulé dès le début. Ainsi, le 10 septembre 1937, le congrès de Bludan déclare « persister à réclamer l'abolition du Mandat et de la promesse Balfour et la substitution à ces deux actes d'un traité à conclure avec la GrandeBretagne $\gg{ }^{1}$.

Dix ans plus tard, les débats de l'assemblée générale extraordinaire des Nations Unies témoignent des mêmes lignes de fracture. Entre-temps, les sionistes ont clairement exprimé leur revendication d'un État. Les responsables du mouvement ont adopté le programme de Biltmore le 11 mai 1942 et l'Agence juive a réclamé, le 22 mai 1945, le contrôle de l'immigration juive et la création d'un État juif. On lit dans le programme de Biltmore :

\footnotetext{
${ }^{1}$ Henry Laurens, La question de la Palestine, T II Une mission sacrée de civilisation, Paris, Fayard, 2002, p. 371.
} 
La conférence demande instamment que les portes de la Palestine soient ouvertes; que l'Agence juive soit chargée de diriger l'immigration en Palestine et soit investie de l'autorité nécessaire pour construire le pays, ce qui comprend la mise en valeur de ses terres vacantes et incultes; et que la Palestine soit constituée en une communauté politique juive intégrée dans l'organisation du nouveau monde démocratique. ${ }^{2}$

De son côté, la Ligue arabe relaie la position défendue par les Arabes de Palestine depuis le début du mandat comme en témoigne cet extrait de lettre envoyé à Ernest Bevin, secrétaire au Foreign Office, le 5 décembre 1946 :

\begin{abstract}
$\mathrm{Au}$ cours des vingt-six années qui ont suivi la Déclaration Balfour, les événements de Palestine ont fourni la preuve que la persécution ne se guérit pas par une persécution nouvelle et que l'ambition de la politique sioniste, celle de voir les sionistes s'emparer de la Palestine, pour y former une majorité grâce à laquelle elle deviendrait le siège d'un État juif, a suscité des troubles graves et donné lieu à des frictions incessantes entre Arabes et Juifs, rendant impossible un compromis entre les deux parties. ${ }^{3}$
\end{abstract}

\title{
Affaire de diplomates
}

Par ailleurs, la Déclaration Balfour donne paradoxalement raison à Theodor Herzl. Cet homme du XIX ${ }^{\mathrm{e}}$ siècle fonde tous ses espoirs sur une percée diplomatique. Loin de se satisfaire d'une installation progressive en Palestine, à l'instar des Amants de Sion et des colonies du baron Rothschild, il veut obtenir une charte de l'Empire ottoman. Il multiplie les initiatives diplomatiques mais n'essuie que des revers.

Vingt ans après la création du mouvement sioniste, les faits lui rendent cependant justice. La présence juive augmente en Palestine dès lors que la Grande-Bretagne publie la Déclaration Balfour et que le mandat y prévoit l'établissement d'un foyer juif. «Le poids démographique, économique, social et politique du Yishouv s'accrut bien plus rapidement que du côté arabe », note ainsi Benny Morris ${ }^{4}$.

\footnotetext{
${ }^{2}$ Cité par Walter Laqueur, Histoire du sionisme, T II, Paris, Tel Gallimard, 1973, p. 790 .

${ }^{3}$ Cité dans Henry Laurens, Le retour des exilés, La lutte pour la Palestine de 1869 à 1997, Paris, Robert Laffont, 1998, p. 619.

${ }^{4}$ Benny Morris, Victimes, Histoire revisitée du conflit arabo-sioniste, Paris, éd. Complexe et CNRS, 2003, p. 124.
} 
Ce développement n'est bien évidemment pas nécessairement inscrit dans la déclaration. Il résulte de plusieurs facteurs, dont la motivation, l'organisation et l'unité de la communauté juive. Quand les Arabes de Palestine éprouvent les pires difficultés à surmonter leurs divisions, la communauté juive crée très rapidement ses instances, véritables institutions pré-étatiques.

En 1917, Londres prépare sa déclaration en liaison étroite avec Washington, revoyant même sa copie à deux reprises pour obtenir l'aval du président américain. La même année, le retrait de la Russie bolchevique laisse toute liberté aux puissances occidentales pour envisager, avant même la fin de la guerre, l'administration des provinces arabes de l'Empire ottoman.

En 1920, lors de la conférence interalliée de San Remo qui prépare le traité de paix avec la Turquie, ces pays européens en arrêtent définitivement les principes et forgent la notion de mandat qu'elles font valider par la Société des Nations en 1922.

Des trois engagements pris par les Britanniques durant la guerre constitution d'un grand royaume indépendant, contrôle européen des anciennes provinces de l'Empire ottoman et soutien à l'établissement d'un foyer national juif -, ils maintiennent les deux derniers, réitérant notamment le soutien à la Déclaration Balfour.

L'article 95 du traité de paix avec la Turquie stipule en effet que :

[...] le mandataire sera responsable de la mise à exécution de la déclaration originairement faite le 2 novembre 1917 par le Gouvernement britannique et adoptée par les autres puissances alliées, en faveur de l'établissement en Palestine d'un foyer national pour le peuple juif. ${ }^{5}$

Il reprend les termes de la Déclaration Balfour et suscite les mêmes lectures contradictoires.

En témoigne la remarque du Président du conseil français Alexandre Millerand :

Le gouvernement français et ses délégués dans les différentes conférences n'ont jamais admis que la Palestine puisse devenir un État sioniste ni qu'un régime sioniste puisse être instauré en Palestine. ${ }^{6}$

\footnotetext{
${ }^{5}$ Henry Laurens, La question de la Palestine, T I L'invention de la Terre sainte, Paris, Fayard, 1999, p. 516.

${ }^{6}$ Ibid.
} 
Tout au long de son administration mandataire, la Grande-Bretagne veille à obtenir l'accord de la Société des Nations sur sa gestion de la Palestine. Si elle perd son empire et se retrouve très affaiblie après la Seconde Guerre mondiale, les États-Unis jouent un rôle qui ne cessera de se développer. Trente ans après la Déclaration Balfour, au début de la guerre froide, c'est parce que les États-Unis et la Russie font, pour des raisons diamétralement opposées, le choix du partage de la Palestine que la question peut être débattue et votée par l'Assemblée générale des Nations Unies.

Ainsi la Déclaration Balfour est-elle la première d'une longue série de décisions diplomatiques - bilatérales ou multilatérales - relatives à la Palestine. Qu'elles émanent d'une grande puissance, de plusieurs ou de l'Organisation des Nations Unies, elles définissent un cadre de coexistence que les peuples juif et arabo-palestinien mettent à mal sur le terrain.

\section{Préserver les communautés de la diaspora}

Les rédacteurs de la Déclaration Balfour veillent ensuite à préciser que le soutien à l'établissement d'un foyer national pour le peuple juif implique clairement : «que rien ne sera fait qui pourrait porter préjudice aux droits civils et religieux des communautés non juives en Palestine, ainsi qu'aux droits et au statut politique dont les Juifs pourraient jouir dans tout autre pays ».

La question des droits des Juifs installés dans d'autres pays semble suffisamment préoccupante pour susciter une éventuelle opposition au mouvement sioniste. Après tout, en 1917, celui-ci est loin d'être majoritaire dans le monde juif et les Juifs, qui étaient souvent persécutés, sont à présent bien intégrés dans les pays d'Europe occidentale et aux États-Unis.

L'émancipation ne paraît pas si ancienne qu'elle soit acquise à jamais. On veut croire que l'élection d'un maire antisémite à Vienne, l'affaire Dreyfus à Paris ou encore la montée de l'antisémitisme en Allemagne ne la remettent pas en cause. Quant à la situation dramatique des Juifs en Europe orientale, on estime généralement qu'elle exige une autre approche.

C'est bien l'interrogation d'Edwin Montagu, secrétaire d'État à l'Inde. Fils d'un Juif orthodoxe, « il voyait avec horreur les sionistes travailler à un édifice qu'il considérait comme un ghetto géant pour tous 
les Juifs du monde », écrit Jean-Pierre Alem ${ }^{7}$. Le ministre britannique reprend les arguments défendus par les assimilationnistes. Cela « aurait pour effet de marquer les Juifs comme des étrangers dans leurs propres pays et de compromettre leur position durement gagnée de citoyens de ces pays $\gg{ }^{8}$.

La question du maintien des droits des Juifs dans les autres pays est en quelque sorte reléguée au second plan lorsque les manifestations d'antisémitisme se multiplient, en Allemagne notamment. Dans les années trente, l'immigration juive en Palestine, qui avait ralenti en raison de la crise économique, repart à la hausse et se maintient à un niveau élevé jusqu'à la promulgation du troisième Livre blanc sur la Palestine le 17 mai 1939. Loin d'apparaître comme un risque pour les communautés persécutées de la diaspora, le sionisme ouvre alors des perspectives.

À la création d'Israël, la question ne se pose plus dans les mêmes termes qu'en 1917. Le développement de l'État ne semble plus du tout compromettre celui des communautés de la diaspora. L'expulsion des Juifs des pays arabes à partir des années cinquante est perçue comme une conséquence du conflit israélo-arabe. Le refus soviétique de permettre l'émigration des Juifs russes s'inscrit, quant à lui, dans la continuité de la politique d'autorités qui leur ont toujours dénié la moindre reconnaissance.

S'il n'est pas question de contraindre les communautés juives de la diaspora, qui se développent parallèlement à l'État juif à l'instar des communautés américaine et française, les responsables du nouvel État invitent les Juifs du monde entier à le soutenir. La déclaration d'indépendance en 1948 affirme :

Nous lançons un appel au peuple juif de par le monde à se rallier à nous dans la tâche d'immigration et de mise en valeur, et à nous assister dans le grand combat que nous livrons pour réaliser le rêve poursuivi de génération en génération : la rédemption d'Israël.

À ce moment-là, les responsables israéliens sont moins sensibles au souci de ne pas remettre en cause le statut des communautés de la diaspora qu'à celui du développement démographique de l'État hébreu. D'ailleurs, par la suite, certains n'hésitent pas instrumentaliser des attentats pour faire pression sur une communauté nationale comme le

\footnotetext{
${ }^{7}$ Jean-Pierre Alem, La Déclaration Balfour aux sources de l'État d'Israël, Bruxelles, Ed. Complexe, 1982, p. 107.

${ }^{8}$ Ibid.
} 
firent Ariel Sharon le 18 juillet 2004 puis Benjamin Netanyahu le 10 janvier 2015, s'attirant les foudres des responsables de la communauté française ${ }^{9}$.

«Immigrez en Israël aussi vite que possible» avait déclaré le premier; "Qu'il laisse la communauté juive française réagir sur les problèmes qui la concernent », avait, par exemple, répondu Théo Klein. Netanyahu de son côté avait dit au lendemain des attentats de janvier 2015 « À tous les Juifs de France, tous les Juifs d'Europe, je vous dis : Israël n'est pas seulement le lieu vers lequel vous vous tournez pour prier, Israël est votre foyer $»{ }^{10}$.

À cet égard, la Déclaration Balfour n'a donc plus d'effets aujourd'hui. La distinction est bien établie et bien perçue entre l'État d'Israël et les différentes communautés juives nationales. Mais, remarque Régine Azria, « sauf événement majeur grave, l'immigration des Juifs en détresse est quasiment tarie. L'aliya est désormais une affaire de choix individuel $[\ldots] »^{11}$.

\section{Une approche rejetée dès le début}

« Rien ne sera fait qui pourrait porter préjudice aux droits civils et religieux des communautés non-juives en Palestine ». Durant le mandat, cette recommandation de la Déclaration Balfour est appliquée. Même s'ils les contrôlent, voire les instrumentalisent comme ils le font à l'occasion de l'élection de Hajj Amin El Husseini comme mufti de Jérusalem le 15 avril 1921, les Britanniques respectent les institutions et les pratiques musulmanes, qu'il s'agisse, par exemple, du Conseil suprême musulman, de l'administration des Waqfs ou encore des lieux saints musulmans.

Au lendemain de la Conférence de San Remo, le gouvernement britannique informe la population des décisions prises :

Conséquemment, l'inclusion de la Déclaration Balfour dans le traité de paix avec la Syrie signifie : a) Que, sous réserve du maintien de l'ordre et de la sécurité

\footnotetext{
9 http://www1.rfi.fr/actufr/articles/055/article_29360.asp Page consultée le 26 août 2017.

10 http://www.lexpress.fr/actualites/1/societe/netanyahu-aux-juifs-de-france-israel-estvotre-foyer_1639413.html Page consultée le 26 août 2017.

${ }^{11}$ Régine Azria, "L'État d'Israël et la diaspora : une relation complexe » dans L'État d'Israël, Alain Dieckhoff (dir), Paris, Fayard, 2008, p. 346.
} 
publique, il n'y aura aucune immixtion dans les coutumes religieuses ni dans les Lieux saints, ni aucune diminution de la liberté de culte $[\ldots]^{12}$

Cette disposition est reprise dans l'article 9 de la charte du mandat :

Le respect du statut personnel des diverses populations et communautés et de leurs intérêts d'ordre religieux sera entièrement garanti. En particulier, le Mandataire exercera le contrôle de l'administration des Wakoufs, en parfaite conformité avec les lois religieuses et la volonté des fondateurs. ${ }^{13}$

La Grande-Bretagne est mise à l'épreuve en août 1929 lorsqu'éclate la crise du Mur occidental. Musulmans et juifs s'accusent mutuellement de violations du statu quo. En juin 1931, la SDN énonce le règlement du Mur occidental, précisant les droits et obligations de chaque communauté ${ }^{14}$.

L'État d'Israël s'inscrit dans la continuité de la Déclaration Balfour. Dans la déclaration d'indépendance, il affirme qu'il « assurera la sauvegarde et l'inviolabilité des Lieux saints et des sanctuaires de toutes les religions ». Il n'en exerce pas moins une surveillance exigeante, pour des raisons de sécurité, comme le montre la récente crise du Mont du Temple.

Cette partie de la Déclaration Balfour est néanmoins sans conteste celle qui suscite le plus tôt de virulentes oppositions et qui l'inscrit dans un contexte chronologique précis, celui de la colonisation. En effet, les rédacteurs se contentent de distinguer les Juifs des autres et ne citent pas les droits politiques, comme le remarque Rashid Khalidi qui évoque « la cage de fer du mandat ${ }^{15}$.

Après les émeutes de 1921 et l'envoi d'une commission d'enquête en Palestine, le gouvernement britannique amorce une première évolution, timide au demeurant puisque, dans le Livre blanc de 1922, il prend acte de l'existence "d'un peuple et d'une langue arabes ». " [Le gouvernement britannique] n'a pas davantage envisagé, comme semble le craindre la Délégation arabe, la disparition ou la subordination de la population, de la langue ou de la culture arabes en Palestine ", lit-on notamment dans ce document ${ }^{16}$.

\footnotetext{
${ }^{12}$ Cité par Henry Laurens, Le retour des exilés, op. cit., p. 297.

${ }^{13}$ Cité par Henry Laurens, La question de la Palestine, T I, op. cit., p. 622.

${ }^{14}$ Cité par Henry Laurens, Le retour des exilés, op. cit., p. 436 et sq.

15 Rashid Khalidi, Palestine Histoire d'un État introuvable, Arles, Actes Sud, 2007, p. 89.

${ }^{16}$ Henry Laurens, La question de la Palestine, T I, op. cit., p. 610.
} 
L'opposition arabe, palestinienne, reste néanmoins intacte. D'ailleurs dans la formulation de la charte, les termes demeurent les mêmes que ceux de la Déclaration Balfour comme le note Henry Laurens :

Le terme de «peuple» n'est utilisé qu'en référence au peuple juif de la Déclaration Balfour. «Population», « habitants» et « indigènes » sont les références en ce qui concerne les gens vivant en Palestine et marquent l'absence de droits politiques en dépit de l'article 22 : la nationalité palestinienne est une réalité pratique qui ne renvoie pas à l'existence d'une nation palestinienne. ${ }^{17}$

Le 11 octobre 1923, Herbert Samuel, le premier haut-commissaire britannique en Palestine, présente aux notables palestiniens un projet d'Agence arabe: «Le Gouvernement de Sa Majesté me demande d'ajouter ce qui suit : Il considère qu'en créant une Agence arabe il fait un grand pas vers la réalisation des désirs de la population arabe [...] ${ }^{18}$.

Cette fois, le vocabulaire a changé, l'approche politique non. Car, selon l'exécutif arabe, elle est contraire à la charte de la SDN :

[...] fondée sur la réalisation des volontés des peuples et la protection des nations opprimées, non sur l'oppression de la liberté des peuples et sur la violence commise pour les forcer à accepter un plan qui les prive de tous leurs droits politiques.

[Et de conclure]

Le plus honorable que pourrait faire le gouvernement de Sa majesté qui appartient à la SDN est d'abolir la promesse Balfour parce qu'elle n'est pas conforme à la charte et à l'esprit de la SDN. ${ }^{19}$

Dans son rapport, la commission Shaw distingue «Juifs et Arabes» et invite le gouvernement à définir «le sens attaché aux passages du Mandat relatifs à la sauvegarde des droits des populations non juives du pays ». En réalité, le ton change avec le premier plan de partage de la Palestine préconisé par lord Peel le 8 juillet 1937 : «Étant donné la façon dont (Arabes de Palestine et Juifs de Palestine) sont associés, sous le régime du Mandat, le libre gouvernement est impraticable pour l'un ou pour l'autre peuple $»^{20}$.

\footnotetext{
${ }^{17}$ Henry Laurens, La question de la Palestine, T I, op. cit., p. 625.

${ }^{18}$ Henry Laurens, La question de la Palestine, T II, op. cit., p. 50.

${ }^{19}$ Ibid.

${ }^{20}$ Cité par Henry Laurens, Le retour des exilés, op. cit., p. 485.
} 
En définitive, en raison des oppositions qu'il soulève, le plan est abandonné le 9 novembre 1938. Il faut attendre dix ans pour que cette option soit reprise, par l'ONU cette fois à la demande du gouvernement britannique. Entre-temps, la publication, le 17 mai 1939, du troisième Livre blanc a été rejetée par les deux parties.

Dans la déclaration d'indépendance, Israël :

[...] invite les habitants arabes du pays à préserver les voies de la paix et à jouer leur rôle dans le développement de l'État sur la base d'une citoyenneté égale et complète et d'une juste représentation dans tous les organismes et les institutions de l'État, qu'ils soient provisoires ou permanents.

Les Arabes israéliens ont ainsi obtenu la citoyenneté mais, pour reprendre un sous-titre de Laurence Louër, "Juifs et Arabes (sont) séparés et inégaux $»^{21}$. L'État a mis en œuvre des politiques publiques discriminatoires tant et si bien que l'auteur parle d'« un processus d'intégration inachevé ». Pour les Palestiniens établis en Cisjordanie et dans la bande de Gaza, qui ne sont pas reconnus comme tels avant les années 1990, l'exercice complet de droits politiques demeure une revendication comme elle l'était au début du $\mathrm{XX}^{\mathrm{e}}$ siècle.

${ }^{21}$ Laurence Louer, «Les citoyens arabes : un processus d'intégration inachevé » dans L'État d'Israël, op. cit., p. 190 et sq. 